\title{
INFORMACION
}

\section{Resoluciones tomadas en el V Congreso del Instituto Internacional de Literatura Iberoamericana}

La Comisión de Iniciativas, que presidió el doctor Francisco Monterde e integraron los doctores Julio Jiménez Rueda y José A. Balseiro, propuso lo siguiente:

I

Dése un voto de cordial simpatía y profundo agradecimiento a la Universidad de Nuevo México, por haber patrocinado este Congreso y por las atenciones que ha tenido para todos los delegados. Exprésese el agradecimiento de la Asamblea al Presidente del Instituto Internacional de Literatura Iberoamericana, Albert R. Lopes; a su Secretario-Tesorero, Marshall R. Nason; a Sabine Reyes Ulibarrí, que lo sustituyó interinamente, y al doctor Arturo Torres-Rioseco, que colaboró con ellos de modo eficaz en la preparación del Congreso. Extiéndase igual manifestación a Sigma Delta $\mathrm{Pi}$, por patrocinar el banquete; a las Universidades y los Colegios que enviaron delegados al mismo Congreso, y especialmente a la Universidad Nacional de México, por su invitación para que el VI Congreso se efectúe en la ciudad de México, en 1953, y por el patrocinio de la Revista Iberonmericana, consistente en el trabajo de impresión de ésta. 
II

Hágase saber a la Uunión Panamericana el interés con que la Asamblea ha visto la publicación de las series "Escritores de América" y "Pensamiento en América", que edita la División de Filosofía, Letras y Ciencias del Departamento de Asuntos Culturales de la propia Unión, y su adhesión a los proyectos que abriga, en cuanto se refiere a la edición de obras difíciles de hallar actualmente.

III

En virtud de la experiencia obtenida a partir del IV Congreso, la comisión propone que se modifique el Art. $6^{\circ}$ de los Estatutos, en los siguientes términos: "La Mesa Directiva estará integrada por:

Un presidente;

Tres Vicepresidentes;

Un Secretario Ejecutivo;

Un Prosecretario;

Un Director General de Publicaciones."

IV

Que se faculte a la Mesa Directiva para que ponga en vigor todas las medidas necesarias para la reorganización del Instituto y designe las comisiones indispensables para los trabajos del Congreso.

Que la nueva Directiva realice una revisión de los acuerdos tomados en asambleas anteriores, con el objeto de que se puedan llevar a la práctica los que sean oportunos.

VI

Que el Instituto estudie la mejor manera de participar en los Centenarios de los nacimientos de Sor Juana Inés de la Cruz, de José 
Martí, de Salvador Díaz Mirón y del doctor Juan Zorrilla de San Martín.

Que el Instituto otorgue cada año, como reconocimiento de los méritos de la obra más destacada, entre las publicadas el año anterior, un premio y el diploma correspondiente, de acuerdo con las bases que la Junta Directiva establezca.

La Comisión Electora y de Sede para el Sexto Congreso del Instituto Internacional de Literatura Iberoamericana, presidida por el doctor Robert M. Duncan e integrada por los doctores Arturo Torres-Rioseco y Francisco Aguilera, hizo las siguientes recomendaciones:

Que la sede del Sexto Congreso sea México.

II

Que las siguientes personas sean designadas para desempeñar los cargos correspondientes:

Presidente: Luis Monguió;

Primer Vicepresidente: Agustín Yáñez;

Segundo Vicepresidente: Enrique Anderson-Imbert;

Tercer Vicepresidente: Benjamín M. Woodbridge, Jr.;

Secretario Ejecutivo: Marshall R. Nason;

Prosecretario: Sabine R. Ulibarrí;

Director General de Publicaciones: Julio Jiménez Rueda. 



\section{Socios y Suscriptores Protectores}

\section{(PERSONAS E INSTITUCIONES)}

Theodore J. Brenner

St. Mary's University

San Antonio 7, Texas, E. U. A.

Prof. Boyd G. Carter

Department of Romance Languages

University of Nebraska

Lincoln, Nebraska, E. U. A.

Claremont College Library

Claremont, California, E. U. A.

John E. Englekirk

Department of Spanish

Tulane University

New Orleans;'Louisiana, E. U.,A.

José Famadas

Box 8

Rego Park, N. Y., E. U. A.

Dr. Max Henríquez Ureña

99-22 67th Road

Forest Hills, Long Island

New York, E. U. A.

Sr. Andrés Horcasitas

Whitney Building
New Orleans 12

Louisiana, E. U. A.

Albert R. Lopes

Box 20

University of New Mexico

Albuquerque, New Mexico,

E. U. A.

University of Michigan

General Library

Ann Arbor, Michigan, E. U. A.

Malcolm D. Mc Lean

723 Woolsey Ave.

Fayetteville, Arkansas, E. U. A.

University of North Carolina Library

Box 690

Chapel Hill, North Carolina, E. U. A.

Pan American Union

Washington, D. C., E. U. A.

Princeton University Library

Princeton, New Jersey, E. U. A. 
Prof. D. F. Ratcliff

Universidad de la República

Department of Romance Lan- Departamento de Literatura Ibeguages roamericana

University of Cincinnati

Campus Station

Cincinnati 21, Ohio, E. U. A.

Facultad de Humanidades y Ciencias

San Antonio Public Library 210 West Market St.

San Antonio, Texas, E. U. A.

Biblioteca-Universidad de Santo Domingo

Ciudad Trujillo

República Dominicana

University of Southern California

Los Angeles 7, California, E. U. A.
Cerrito 73

Montevideo, Uruguay

University of Washington $\mathrm{Li}-$ brary

Seattle 5, Washington, E. U. A.

Wellesley College Library

Wellesley, Mass., E. U. A.

Williams College Library

Williamstown, Massachusetts, E. U. A. 